\title{
UM ESTUDO SOBRE A UTILIZAÇÃO DA MODELAGEM DE EQUAÇÕES ESTRUTURAIS NA PRODUÇÃO CIENTIFICA NAS ÁREAS DE ADMINISTRAÇÃÓ E SISTEMAS DE INFORMAÇÃO
}

\author{
A STUDY ON THE USE OF STRUCTURAL EQUATION \\ MODELING IN SCIENTIFIC PRODUCTION IN THE FIELDS OF \\ MANAGEMENT AND INFORMATION SYSTEMS
}

Data de submissão: 08-04-2013 Aceite: 02-04-2015

Ronielton Rezende Oliveira ${ }^{1}$ Marlon Finelli Avendanha Marinho²

Alexandre Teixeira Dias ${ }^{3}$

\section{RESUMO}

Este artigo apresenta um estudo da produção acadêmica no período de 2008 a 2012 em relação as pesquisas que utilizaram a Modelagem de Equações Estruturais como método quantitativo para análise de dados. A amostra de 44 artigos foi analisada por meio da metodologia de avaliação de dimensões de qualidade sugerida por Hoppen, Lapointe e Moreau (1996) adaptada para este estudo. Foram extraídos artigos indexados na base de dados Scientific Periodical Electronic Library e de revistas cientificas pré-selecionadas da área de Administração e Sistemas de Informação com o uso de palavras chaves. Estes tiveram seu conteúdo analisado em função das variáveis da estrutura de pesquisa: natureza, questão de pesquisa, desenho de pesquisa, instrumento de medida e coleta de dados, validade dos construtos, análise dos dados e apresentação dos resultados. Este estudo identificou nos artigos analisados um rigor moderado na preparação e instrumentalização das pesquisas, ao considerar os aspectos de qualidade esperados para uma pesquisa quantitativa.

Palavras chave: Produção Acadêmica; Modelagem de Equações Estruturais; Avaliação de Pesquisas Quantitativas; Mapeamento Bibliométrico; Técnicas Estatísticas.

\footnotetext{
1 Possui Graduação em Ciência da Computação pela Universidade Vale do Rio Verde de Três Corações, UNINCOR. Especialização em Criptografia e Segurança em Redes pela Universidade Federal Fluminense, UFF. Mestrado profissional em Sistemas de Informação e Gestão do Conhecimento pela Universidade FUMEC, FUMEC. Doutorado em andamento em Administração pela Universidade FUMEC, FUMEC. Belo Horizonte. Minas Gerais. Brasil. E-mail: ronielton@fumec.edu.br

2 Possui Graduação em Gestão da Tecnologia da Informação pela Universidade FUMEC, FUMEC. Mestrado em Administração pela Universidade FUMEC, FUMEC. Belo Horizonte. Minas Gerais. Brasil. E-mail: finellimarlon@gmail.com

3 Possui Graduação em Administração pela Universidade Federal de Minas Gerais, UFMG. Especialização em Gestão de Negócios pela Universidade Federal de Minas Gerais, UFMG. Mestrado em Administração pela Universidade Federal de Minas Gerais, UFMG. Doutorado em Administração pela Universidade Federal de Minas Gerais, UFMG. Belo Horizonte. Minas Gerais. Brasil. E-mail: alexandre.tdias@fumec.br
} 


\section{ABSTRACT}

This article presents a study of academic production in the period 2008-2012 in relation to the researches using Structural Equation Modeling as a quantitative method for data analysis. A sample of 44 articles was analyzed by means of the evaluation methodology of quality dimensions suggested by Hoppen, Lapointe and Moreau (1996) adapted for this study. We extracted articles indexed in the database Scientific Periodical Electronic Library and journals pre-selected area of Administration and Information Systems with the use of keywords. These had their content analyzed according to the variables of the research structure: nature, research question, research design, measurement instrument and data collection, validity of the constructs, data analysis and presentation of results. This study identified in the articles analyzed a moderate accuracy in the preparation and manipulation of research when considering the quality aspects expected for a quantitative research.

Keywords: Academic Production; Structural Equation Modeling; Evaluation of Quantitative Research; Bibliometric Mapping; Technical Statistics.

\section{INTRODUÇÃO}

No "mundo real", os modelos ajudam a entender se existem e como se dão as relações entre pessoas, fatos, ideias, conceitos e outros. Nota-se que as técnicas quantitativas têm sido aplicadas para modelar a realidade e buscar a previsibilidade. A Modelagem de Equações Estruturais (MEE) é um método estatístico de segunda geração (HAIR JR. et al., 2013), que destaca-se como uma ferramenta que possibilita desenvolver uma análise confirmatória ao verificar se os dados coletados, mostram evidências de que realmente se comportam como o modelo teórico em função da teoria subjacente ao problema de pesquisa.

Nesta perspectiva, a MEE é um método adequado para a pesquisa social, sendo considerado o mais avançado do ponto de vista da sua capacidade de articulação de variáveis sociais, o qual é respeitado no meio acadêmico como uma abordagem estatística para testar hipóteses a respeito de relações entre variáveis (CODES, 2005). Em linhas gerais, essa técnica de análise quantitativa multivariada, consiste em modelar a realidade para testar a plausibilidade de um modelo construído com base em uma teoria sobre o fenômeno em estudo, ao permitir que diversas variáveis sejam correlacionadas simultaneamente, de modo que as múltiplas relações possíveis sejam apreciadas.

Assim, a MEE é uma técnica avançada de tratamento e análise estatística de dados, adequada ao estudo de fenômenos complexos, que envolvem múltiplos fatores e caracteriza-se pela sua capacidade de especificar, estimar e testar relações hipotéticas entre um grupo de variáveis (GOSLING; GONÇALVES, 2003). Portanto, o problema de pesquisa que delimita este trabalho é: Quais critérios devem ser considerados para a apresentação e discussão dos resultados em uma pesquisa quantitativa com tratamento dos dados por meio da análise estatística multivariada que utiliza a Modelagem de Equações Estruturais?

Deste modo Hoppen, Lapointe e Moreau (1996), sugerem que na falta de uma fonte confiável e única que indique quais critérios escolher, torna-se essencial a realização de uma síntese das diversas fontes de referência disponíveis, de modo a abranger todas as dimensões da qualidade de uma pesquisa e evitar que alguns critérios sejam esquecidos ou negligenciados. Por isso, o objetivo deste estudo foi recuperar informações de artigos científicos que adotaram a Modelagem de Equações Estruturais (MEE), para construção de modelos que refletem as associações entre os conceitos teóricos nos respectivos estudos, com a finalidade de mensurar os critérios utilizados naqueles trabalhos e relatar os resultados encontrados ao capturar as dimensões de qualidade necessárias em um estudo quantitativo com uso da MEE no tratamento dos dados. 
Este artigo está organizado em cinco partes, além desta introdução. A segunda parte, apresenta uma revisão de literatura sobre o método de avaliação para pesquisas proposto por Hoppen, Lapointe e Moreau (1996) e uma breve introdução a Modelagem de Equações Estruturais. Na terceira parte, classifica-se o estudo quanto a metodologia e apresentam-se as considerações a respeito do processo de escolha da amostra. Na quarta parte, os dados são analisados em função das dimensões de pesquisa oriundas do método de avaliação adaptado para este estudo: natureza, questão de pesquisa, desenho de pesquisa, instrumento de medida e coleta de dados, validade dos construtos, análise dos dados e apresentação dos resultados. Por fim, na quinta parte, considerações finais, são apresentadas as observações a respeito do estudo, as limitações e as sugestões para pesquisas futuras.

\section{REFERÊNCIAL TEÓRICO}

\subsection{Dimensões para Avaliação de Pesquisas Quantitativas}

Existem muitas ocasiões em que um pesquisador deve julgar a qualidade de uma pesquisa produzida em seu domínio de conhecimento. Mas acontece, que é difícil julgar o valor de uma pesquisa ou de um artigo científico, e isto por três razões essenciais: a falta de critérios precisos de avaliação, a falta de detalhes relativos aos procedimentos de pesquisa e impossibilidade de utilizar os mesmos critérios em função do método escolhido (HOPPEN; LAPOINTE; MOREAU, 1996).

A escolha do método de pesquisa depende do objeto da pesquisa. Por isso, Hoppen, Lapointe e Moreau (1996), apontam que na concepção, os autores negligenciam certos detalhes essenciais, o que na posterior avaliação da pesquisa, torna difícil apreciar o estudo feito. Assim, a falta de informações sobre o enunciado da questão de pesquisa, os detalhes sobre os métodos de validação, as informações relativas à amostra, as delimitações do estudo, entre outras, emergem como um potencial obstáculo a tarefa de avaliação, além de induzir a questionamentos sobre a qualidade do trabalho.

Porém, uma vez estabelecidos critérios para a avaliação, estes podem ser usados na concepção e no desenvolvimento de novos estudos. Nesta linha de pensamento, Hoppen, Lapointe e Moreau (1996), procuraram sintetizar as principais formas de pesquisa e agrupar alguns critérios de análise, que podem ser considerados relevantes para a avaliação e para o desenvolvimento de artigos em Sistemas de Informação. As formas e os critérios foram organizados pelos mesmos autores para o processo de investigação científica nos métodos "por enquete, experimental e qualitativos" em "grades de análise" para "facilitar a avaliação das pesquisas" (HOPPEN; LAPOINTE; MOREAU, 1996, p. 4). Especificamente, a grade de análise para o método "por enquete", concebido para avaliação de pesquisas estruturadas com o uso de questionários foram organizadas em torno de um conjunto de critérios agrupados em categorias, a saber: a pertinência do método escolhido, o objeto da pesquisa, o desenho de pesquisa, os instrumentos de medida e a coleta de dados, a validade do construto, a análise dos dados, a apresentação dos resultados e o estilo do artigo.

O "método de pesquisa", é dependente dos conhecimentos, das aptidões e das preferências do pesquisador e deve representar a escolha por uma área de pesquisa predominante, quantitativa ou qualitativa. Isto, direciona a verificação da adequação entre o método, o problema de pesquisa e a hipótese. A "natureza", relaciona o método adotado, quanto aos fins para a condução da pesquisa. A "questão de pesquisa", corresponde a várias dimensões do estudo. Quando da sua análise é preciso interrogar-se sobre os objetivos do artigo e sua pertinência. $O$ enunciado deve constar no texto, ser claro, preciso e fácil de identificar. Este, também permite identificar a(s) teoria(s) utilizadas para fundamentar o estudo. Além disso, de modo implícito ou explícito, a questão de pesquisa pode descrever o modelo de pesquisa em que se baseia, ao 
enunciar as hipóteses que estão na origem das suas indagações. O "desenho de pesquisa", pode ser definido como a sequência lógica que liga os dados empíricos à questão de pesquisa, aos resultados e as conclusões (HOPPEN; LAPOINTE; MOREAU, 1996).

A dimensão "instrumento de medida e coleta de dados", considera os conceitos relacionados aos procedimentos para o desenvolvimento de instrumentos de medida, definição da amostra e atividade de coleta de dados. A "validade dos construtos", está diretamente relacionada à questão do que o estudo, por meio do instrumento de pesquisa está realmente medindo. O termo significa que a medida avalia a magnitude e a direção de todas as características, e somente das características do construto que ela pretende avaliar de maneira genérica, ao analisar a confiabilidade e a validade das medidas. A "análise de dados", é a dimensão que julga a pertinência e a qualidade de aplicação dos métodos utilizados, em função dos dados qualitativos ou quantitativos, e da escolha de uma técnica de análise apropriada. Por fim, a "apresentação dos resultados", demonstra as condições de interpretação dos achados para o leitor, ao se fazer a ligação com a teoria que serviu para o desenvolvimento da pesquisa por meio de um texto claro, bem organizado e bem estruturado (HOPPEN; LAPOINTE; MOREAU, 1996).

Portanto, para este estudo foi adaptado o método "por enquete" da proposta de Hoppen, Lapointe e Moreau (1996), para a seleção e avaliação das dimensões de qualidade de artigos, as quais emergem como uma preocupação dos profissionais que são usuários da informação (SOLA; BONACIM, 2013, p. 56). Especialmente, aquelas relacionadas as pesquisas que adotaram a Modelagem de Equações Estruturais para tratamento dos dados coletados por questionários estruturados na área de Administração e Sistemas de Informação.

\subsection{Modelagem de Equações Estruturais}

A Modelagem de Equações Estruturais (MEE) é uma das técnicas de análise estatísticas mais úteis e avançadas que surgiram nas ciências sociais nas últimas décadas. É uma classe de técnicas de análise multivariada, que combina aspectos de análise fatorial e de regressão, permitindo ao pesquisador examinar simultaneamente, as relações entre as variáveis observáveis e as variáveis latentes, bem como entre variáveis latentes (HAIR JR. et al., 2013).

Nota-se, que as técnicas quantitativas têm sido aplicadas para modelar a realidade e buscar a previsibilidade. Codes (2005), afirma que a MEE é um método adequado ao estudo de fenômenos complexos, que envolvem múltiplos fatores e caracteriza-se pela sua capacidade de especificar, estimar e testar relações hipotéticas entre um grupo de variáveis. Deste modo, a MEE é uma ferramenta que possibilita verificar se os dados coletados mostram evidências de que realmente se comportam como o modelo idealizado e testado, e deve ser usada de acordo com o problema de pesquisa previamente definido (GOSLING; GONÇALVES, 2003).

Em linhas gerais, essa técnica de análise quantitativa multivariada consiste em testar a plausibilidade de um modelo construído com base em uma teoria sobre o fenômeno estudado. Pois, permite que diversas variáveis sejam correlacionadas simultaneamente, de modo que, as múltiplas relações possíveis sejam apreciadas, uma vez que, a proposta central é que se construam modelos que possam refletir as associações entre conceitos, tais como descritos pela teoria (CODES, 2005).

Para Zwicker, Souza e Bido (2008), a MEE é em si a técnica que permite a combinação de um modelo de mensuração a um modelo estrutural, avaliados simultaneamente. Há, entretanto, mais de um método para a estimação dos coeficientes de mensuração e estruturais dos modelos. Hair Jr. et al. (2013) corrobora que, embora existam muitos métodos para a realização da MEE, o método mais amplamente utilizado é certamente o Covariance-based (MEE-CB). Percebe-se, 
que o uso do Partial Least Squares (MEE-PLS) aumentou exponencialmente em uma variedade de disciplinas, com o reconhecimento de que as características metodológicas distintas da MEE-PLS tornam-na uma alternativa viável a abordagem mais popular MEE-CB (HAIR JR. et al., 2013).

Em relação ao uso, a modelagem exploratória (MEE-PLS) desenvolve a teoria, enquanto, a modelagem confirmatória (MEE-CB) testa a teoria (ZWICKER; SOUZA; BIDO, 2008; HAIR JR. et al., 2013). O que confirma, que a MEE é usada tanto para explorar ou confirmar (ou rejeitar) a teoria. De fato, a MEE-PLS é o método preferido quando o objetivo da pesquisa é o desenvolvimento de teoria e a explicação da variância com previsão dos construtos. Especificamente, a MEE-PLS tem várias vantagens sobre a MEE-CB em muitas situações comumente encontradas na pesquisa em ciências sociais, por exemplo, quando as amostras são pequenas, os dados não são distribuídos normalmente, ou quando são estimados modelos complexos, com muitos indicadores e relacionamentos no modelo (HAIR JR. et al., 2013).

Este é descrito por um modelo de medidas, também chamado de modelo exterior, relacionando as variáveis observadas nas variáveis latentes correspondentes, e por um modelo estrutural, também chamado de modelo interior, relacionando as variáveis latentes endógenas em outras variáveis latentes, que podem ser endógenas e exógenas (MENDES, 2006). Ou seja, a MEE-PLS usa os dados disponíveis para estimar o caminho dos relacionamentos no modelo com o objetivo de minimizar os erros dos construtos endógenos. Em outras palavras, calcula os coeficientes que maximizam os valores do $\mathrm{R}^{2}$ (coefficient of determination) dos construtos endógenos. Portanto, o path model consiste de dois elementos (HAIR JR. et al., 2013, p. 12), o primeiro componente é o modelo estrutural que mostra as relações entre os construtos latentes. $O$ segundo componente é o modelo estrutural, que compreende o modelo de mensuração. Estes incluem as relações preditivas unidirecionais entre cada construto latente e seus indicadores observados associados (HAIR; RINGLE; SARSTEDT, 2011).

Os caminhos dos relacionamentos no modelo são desenvolvidos com base na teoria. Eles consistem de diagramas que são usados para mostrar visualmente as hipóteses e as relações variáveis que são examinados quando a MEE é utilizada. Então, a MEE-PLS é considerada uma abordagem Variance-based (HAIR JR. et al., 2013). Em outras palavras, o modelo estrutural é que define a relação entre as variáveis latentes exógenas e endógenas. Assim sendo, o modelo estrutural é a representação visual que específica quais variáveis latentes (exógena ou independente) influenciam direita ou indiretamente mudanças nos valores da outra variável latente (endógena ou dependente).

A MEE-PLS tem menores exigências em relação ao tamanho da amostra e à distribuição dos dados, quando comparado a MEE-CB (ZWICKER; SOUZA; BIDO, 2008), e funciona de forma eficiente com tamanhos de amostra pequenos e modelos complexos, pois, faz com que praticamente não existam suposições com os dados da amostra (HAIR JR. et al., 2013), ou seja, por não ter suposições quanto à distribuição das variáveis, não há necessidade de transformação dos indicadores (ZWICKER; SOUZA; BIDO, 2008).

Por fim, resta saber que a MEE, vista como um método de pesquisa e não como uma técnica de análise de dados, envolve atividades e decisões que incluem: a escolha do referencial teórico a ser utilizado; elaboração do modelo estrutural (que especifica a relação entre os construtos) e do modelo de mensuração (especificação dos indicadores para mensurar os construtos ou variáveis latentes) que, às vezes, envolve a construção de instrumentos para a coleta de dados; a coleta de dados propriamente dita; o teste do modelo e; finalmente, a interpretação dos resultados à luz da teoria que foi usada para justificá-lo (BIDO et al., 2012). 


\section{METODOLOGIA}

Este estudo é uma pesquisa quantitativa caracterizada como descritiva, uma vez que se estabeleceu como objetivo, identificar, classificar e analisar (MALHOTRA, 2012), os trabalhos acadêmicos que utilizaram a Modelagem de Equações Estruturais, em um universo de 10.175 artigos científicos publicados na biblioteca Scientific Periodicals Eletronic Library (SPELL) e em uma seleção de revistas cientificas da área de Administração e Sistemas de Informação (RASI) no período de 2008 a 2012. O corte temporal que delimitou a seleção dos trabalhos foi definido em função da catalogação existente na biblioteca SPELL, a qual durante a coleta e obtenção dos artigos em dezembro de 2012, apenas indexava artigos científicos publicados a partir de 2008. Também é uma pesquisa bibliométrica, pois permite obter conhecimento em uma área acadêmica, e serve, entre outras funções, para demonstrar características relacionadas à produção científica desta área (ANDRADE-JÚNIOR; CERANTO, 2013; SOLA; BONACIM, 2013).

Iniciada em 2012, a biblioteca eletrônica SPELL da Associação Nacional de Pós-Graduação e Pesquisa em Administração (ANPAD) é um sistema repositório de indexação, pesquisa e disponibilização gratuita de produção técnico-científica das áreas de Administração, Contabilidade e Turismo. As revistas RASI selecionadas com estrato no Sistema Qualis da Coordenação de Aperfeiçoamento de Pessoal de Nível Superior (CAPES), igual ou superior a B2 tem como área de interesse a produção cientifica da área de Administração e Sistemas de Informação e foram acessadas por meio do endereço eletrônico dos periódicos: Revista de Administração Contemporânea, Revista RAC Eletrônica, Brazilian Administration Review, Revista de Administração e Contabilidade Base, Revista RAE eletrônica, Journal of Computer Sciences, Revista Ciência da Informação e Jornal of Technology Management \& Innovation.

Desta forma, os artigos científicos coletados na base de dados da biblioteca SPELL e nas revistas RASI são os objetos empíricos de estudo em questão neste trabalho, para selecionar e analisar o conteúdo daqueles, nos quais a técnica de análise de dados foi a Modelagem de Equações Estruturais. Para tanto, seguiu-se uma estratégia qualitativa em um procedimento de pesquisa bibliográfica com a análise do conteúdo de cada um dos artigos obtidos. A etapa de seleção dos artigos consistiu na pesquisa dos artigos nas bases de dados, por meio do uso de "palavras chaves" nos idiomas português e inglês. A quantidade de trabalhos localizados é apresentada no quadro 1.

Quadro 1 - Palavras Chave e Quantidade de Artigos

\begin{tabular}{|c|c|c|}
\hline & Biblioteca SPELL & Revistas RASI \\
\hline MEE & 00 & 03 \\
\hline Modelagem de Equações Estruturais & 10 & 07 \\
\hline Equações Estruturais & 09 & 11 \\
\hline SEM & 00 & 05 \\
\hline Structural Equation Modeling & 18 & 21 \\
\hline Structural Equation & 02 & 21 \\
\hline TOTAL & 39 & 68 \\
\hline
\end{tabular}

Fonte: dados da pesquisa, 2013.

A segunda etapa consistiu na análise do título do artigo, por isso, a quantidade de trabalhos únicos foi reduzida a 51 artigos para a análise, visto terem sido descartados aqueles duplicados. A terceira etapa consistiu na catalogação dos artigos, de acordo com os dados de interesse, que serão discutidos na quarta parte deste artigo. A ficha de catalogação resumida é apresentada no quadro 2 . 
Quadro 2 - Ficha de Catalogação Resumida

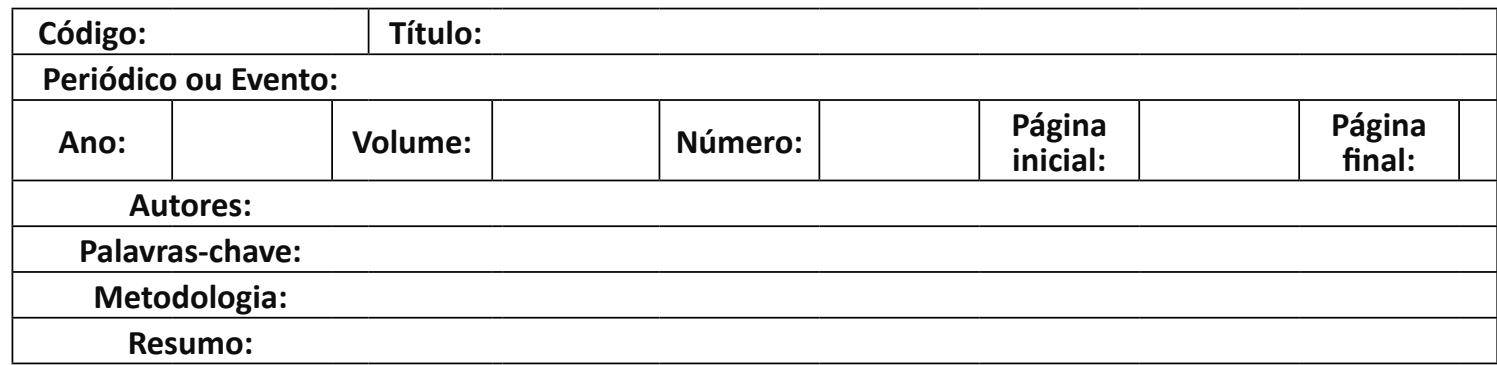

Fonte: elaborado pelos autores.

Dos artigos selecionados, após o preenchimento da ficha de catalogação foram descartados 7 artigos, uma vez estes serem falso-positivos, pois não utilizavam o método de Modelagem de Equações Estruturais para a análise dos dados. Portanto, a amostra deste estudo é composta por 44 artigos, os quais foram analisados de acordo com a metodologia proposta por Hoppen, Lapointe e Moreau (1996), nos aspectos adaptados para este estudo: a) natureza, b) questão de pesquisa, c) desenho de pesquisa, d) instrumento de medida e coleta de dados, e) validade dos construtos, f) análise dos dados, e g) apresentação dos resultados.

\section{RESULTADOS E ANÁLISE}

Este estudo tem o foco de avaliação quantitativa do conteúdo dos artigos nas dimensões de qualidade esperadas em produções acadêmicas provenientes de pesquisas Quantitativas. Foram analisados 44 trabalhos da área de Administração e Sistemas de Informação, que utilizaram a Modelagem de Equações Estruturais para tratamento dos dados entre os anos de 2008 a 2012, a quantidade de trabalhos por ano de publicação é exibida na figura 1.

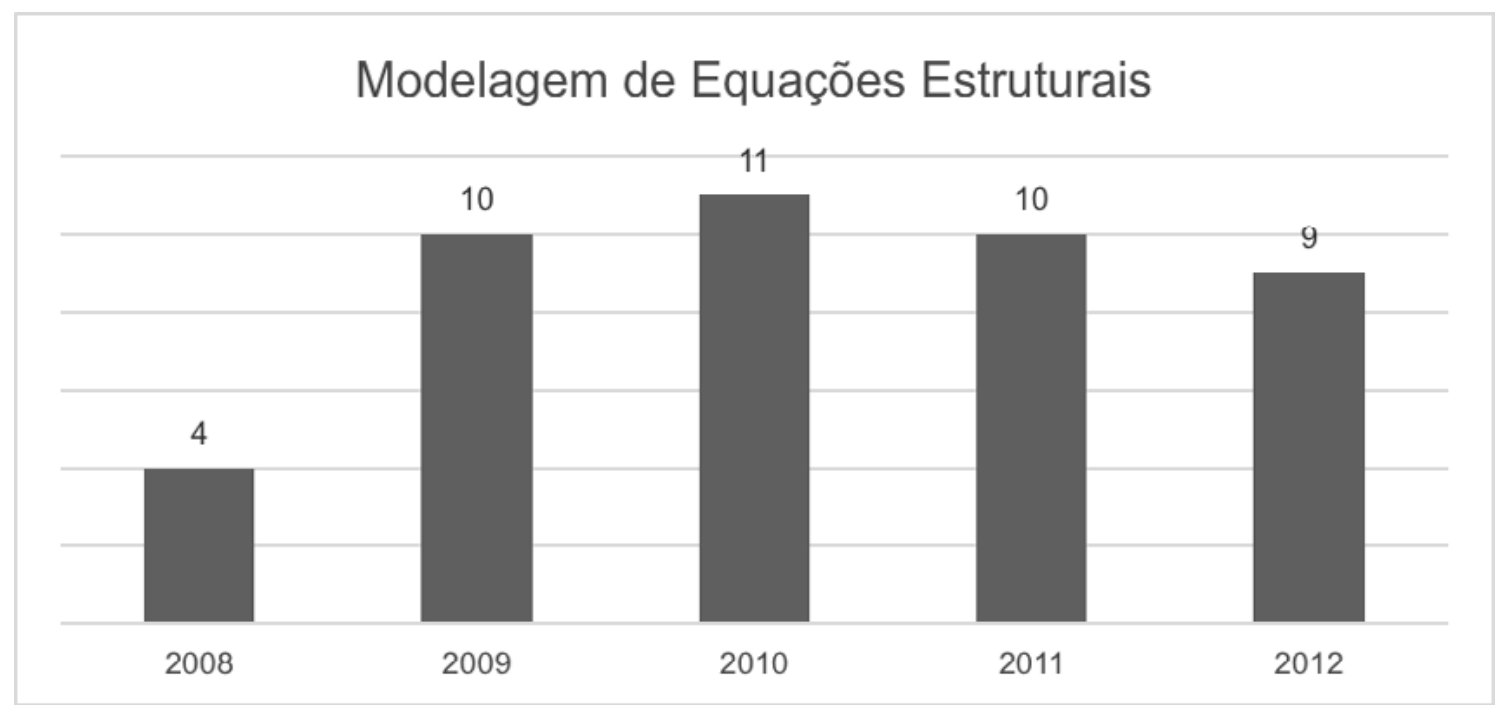

Figura 1 - Quantidade de Trabalhos Publicados por Ano Fonte: dados da pesquisa, 2013.

O idioma preferido nas obras analisadas é o português, 12 artigos foram redigidos no idioma inglês, destes, dois foram publicados em periódicos internacionais (Austrália e Chile). É importante destacar os periódicos Brazilian Administration Review (BAR) e Revista de Adminis- 
tração Contemporânea (RAC), como os que mais publicaram artigos no período considerado. A relação completa dos artigos analisados é apresentada no apêndice 1 . O quadro 3 sumariza os periódicos e a quantidade de trabalhos publicados.

Quadro 3 - Publicação por Periódico

\begin{tabular}{|l|l|l|l|}
\hline Brazilian Administration Review & 8 & Revista Alcance & 1 \\
\hline Revista de Administração Contemporânea & 8 & Revista Brasileira de Finanças & 1 \\
\hline Revista de Administração Mackenzie & 4 & Revista Contabilidade \& Finanças & 1 \\
\hline Revista de Administração & 3 & Revista de Administração de Empresas & 1 \\
\hline $\begin{array}{l}\text { Revista de Administração e Contabilidade } \\
\text { Base }\end{array}$ & 2 & Revista de Administração e Inovação & 1 \\
\hline REAd. Revista Eletrônica de Administração & 2 & Revista de Administração Pública & 1 \\
\hline Revista de Economia e Administração & 2 & Revista de Gestão & 1 \\
\hline Brazilian Business Review & 1 & $\begin{array}{l}\text { Revista do Mestrado em Administração e Desenvol- } \\
\text { vimento Empresarial }\end{array}$ & 1 \\
\hline Journal of Computer Science & 1 & Revista Economia \& Gestão & 1 \\
\hline $\begin{array}{l}\text { Journal of Information Systems and Techno- } \\
\text { logy Management }\end{array}$ & 1 & Revista Eletrônica de Ciência Administrativa & 1 \\
\hline $\begin{array}{l}\text { Journal of Technology Management \& Inno- } \\
\text { vation }\end{array}$ & 1 & Turismo em Análise & 1 \\
\hline
\end{tabular}

Fonte: dados da pesquisa, 2013.

Na sequência, são exibidos os resultados dos artigos analisados, os quais estão agrupados em dimensões de acordo com o conjunto de critérios adaptados da metodologia proposta por Hoppen, Lapointe e Moreau (1996), que devem ser considerados como itens necessários para apresentação e discussão dos resultados de uma pesquisa quantitativa com tratamento dos dados por meio da análise estatística multivariada que utiliza a Modelagem de Equações Estruturais.

\subsection{Natureza}

Esta dimensão, identifica o objetivo geral do estudo, ao classificar o objeto de investigação nos grandes grupos de pesquisa quanto aos fins. Os resultados são apresentados na tabela 1.

Tabela 1 - Dimensão Natureza

\begin{tabular}{|c|c|c|c|c|}
\hline & & & Ocorrência & $\begin{array}{l}\text { Percen- } \\
\text { tual }\end{array}$ \\
\hline \multirow{3}{*}{ 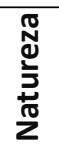 } & \multirow{3}{*}{ Natureza da Pesquisa } & Exploratória & 31 & $65 \%$ \\
\hline & & Descritiva & 16 & $33 \%$ \\
\hline & & Causal & 1 & $2 \%$ \\
\hline
\end{tabular}

Fonte: dados da pesquisa, 2013.

Predomina nos artigos analisados a pesquisa exploratória, a qual estabelece critérios, métodos e técnicas para elaboração, ao fornecer informações sobre o objeto da pesquisa com formulação de hipóteses de modo a aprofundar os conceitos preliminares do pesquisador. Outra parte dos trabalhos é classificada por seus autores como descritivos, nestes o foco é o estudo, a análise, as situações, as opiniões, os comportamentos, o registro e a interpretação dos fatos da população analisada. Por fim, apenas um trabalho registrou os fatos, para analisa-los, interpreta -los e identificar suas causas com o teste da teoria subjacente e suas relações causais. Portanto, considerando a coleta de dados a fim de aprofundar o conhecimento, ou mesmo a intenção de compreender o comportamento dos dados em um determinado contexto, é nítido que o uso da 
modelagem exploratória (MEE-PLS) ou da modelagem confirmatória (MEE-CB), é pertinente aos estudos, uma vez a primeira, no caso da pesquisa exploratória ser utilizada para desenvolver a teoria, e a segunda, no caso da pesquisa descritiva ou causal ser utilizada para testar a teoria. De fato, isto não é restritivo e a MEE-PLS ou a MEE-CB podem ser utilizadas em qualquer natureza de pesquisa, desde que a escolha da técnica para tratamento dos dados esteja amparada pelo problema de pesquisa.

\subsection{Questão de Pesquisa}

Esta dimensão, identifica os aspectos que caracterizam a estrutura de formulação do problema de pesquisa, mais precisamente, explica os objetivos do artigo e sua pertinência ao apresentar a contribuição de investigação para o domínio do conhecimento. Os resultados são apresentados na tabela 2.

Tabela 2 - Dimensão Questão de Pesquisa

\begin{tabular}{|c|c|c|c|c|}
\hline & & & Ocorrência & $\begin{array}{c}\text { Percen- } \\
\text { tual }\end{array}$ \\
\hline \multirow{9}{*}{ 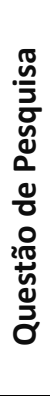 } & \multirow{2}{*}{ Justificativa } & Sim & 31 & $70 \%$ \\
\hline & & Não & 13 & $30 \%$ \\
\hline & \multirow{3}{*}{ Teorias de Base } & Aprofundada & 17 & $39 \%$ \\
\hline & & Superficial & 27 & $61 \%$ \\
\hline & & Ausente ou Não Relacionada & 0 & $0 \%$ \\
\hline & \multirow{2}{*}{ Objetivo / Questão de Pesquisa } & Sim & 41 & $93 \%$ \\
\hline & & Não & 3 & $7 \%$ \\
\hline & \multirow{2}{*}{ Hipóteses / Pressupostos de Base } & Sim & 32 & $73 \%$ \\
\hline & & Não & 12 & $27 \%$ \\
\hline
\end{tabular}

Fonte: dados da pesquisa, 2013.

Ao formular o problema de pesquisa, a maioria dos autores apresentaram a justificativa de escolha do tema. O objetivo é nítido, ou se é possível inferir no texto de $93 \%$ dos trabalhos. Por sua vez, quando pertinentes, as hipóteses que foram testadas com a MEE acompanharam os modelos propostos em 32 trabalhos. Percebe-se, uma preocupação dos pesquisadores em aprofundarem as teorias de base nos seus estudos. Isso significa, definir com clareza qual o referencial teórico que dará sustentação para que a pesquisa seja conduzida a luz da teoria, principalmente, se o assunto é incipiente e ainda não há respostas concretas. Entretanto, é preocupante que em $61 \%$ dos artigos, a teoria foi apresentada de modo superficial, isto, pode influenciar e permitir a contestação do modelo proposto pelos estudos, uma vez que o modelo teórico e seus construtos, ao serem analisados com a MEE, devem estar fortemente relacionados e serem descritos pela teoria.

\subsection{Desenho de Pesquisa}

Esta dimensão, identifica os principais elementos para o desenvolvimento dos processos de investigação, considerados previamente no planejamento operacional da pesquisa, os quais definem a sequência lógica que liga os dados empíricos à questão de pesquisa, os resultados e as conclusões do estudo. Os resultados são apresentados na tabela 3. 
Tabela 3 - Dimensão Desenho de Pesquisa

\begin{tabular}{|c|c|c|c|c|}
\hline \multirow{13}{*}{ 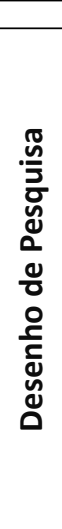 } & & & Ocorrência & Percentual \\
\hline & \multirow{2}{*}{ Modelo de Pesquisa } & Sim & 36 & $82 \%$ \\
\hline & & Não & 8 & $18 \%$ \\
\hline & \multirow{2}{*}{ Tipo de Estudo } & Longitudinal & 22 & $50 \%$ \\
\hline & & Corte Transversal & 22 & $50 \%$ \\
\hline & \multirow{2}{*}{ Mix de Métodos } & Sim & 25 & $57 \%$ \\
\hline & & Não & 19 & $43 \%$ \\
\hline & Objeto da Investigação & \multicolumn{3}{|c|}{ Tabela 4} \\
\hline & Sujeito da Investigação & \multicolumn{3}{|c|}{ Tabela 5} \\
\hline & \multirow{2}{*}{ Operacionalização das Variáveis } & Sim & 32 & $73 \%$ \\
\hline & & Não & 12 & $27 \%$ \\
\hline & \multirow{2}{*}{ Descrição dos Procedimentos Metodológicos } & Sim & 44 & $100 \%$ \\
\hline & & Não & 0 & $0 \%$ \\
\hline
\end{tabular}

Fonte: dados da pesquisa, 2013.

Nota-se que existe uma grande preocupação dos pesquisadores quanto a forma do estudo. O modelo de pesquisa adotado para construir os relacionamentos, entre as variáveis observadas no estudo, que são confrontados com a base teórica, ou uma descrição mais ampla do fenômeno estudado, está bem descrito na maioria dos trabalhos. Em relação à classificação do tipo de estudo, em função do corte temporal persiste o equilíbrio nos trabalhos. Ou seja, metade dos trabalhos analisados utilizou o estudo longitudinal para avaliar um processo dinâmico, implicando numa mudança ocorrida no tempo, com o objetivo de estudar o efeito de uma ou mais variáveis; e a outra metade realizou um estudo de corte transversal com coleta dos dados, em um momento preciso do tempo, com o objetivo de procurar associações comuns entre as variáveis.

Para preparação do modelo, foram utilizados diversos métodos para instruir a questão de pesquisa. As variáveis foram operacionalizadas em 32 artigos (apresentação das fórmulas de cálculo e/ou instrumentos de coleta de dados). Todos os artigos analisados descreveram os procedimentos metodológicos adotados para a preparação, execução e análise dos dados nas pesquisas, o que de fato viabiliza a replicação do estudo. $O$ objeto da investigação pode ser agrupado em oito categorias de acordo com a similaridade nos trabalhos analisados. Assim, qualidade, representa as pesquisas com foco na percepção e satisfação; valor empresarial, significa o conjunto de benefícios que uma organização pretende entregar; mercado, aponta a orientação para o relacionamento com o consumidor e a retenção de clientes; aprendizado, indica o desenvolvimento de competências e o seu relacionamento com o desempenho; valor pessoal, suscita os traços de personalidade dos indivíduos, que são responsáveis pela idealização, socialização, satisfação e cooperação; tecnologia, direciona o uso de sistemas de informação; desempenho, é o modo de mensuração de performance; e metodologia, a forma e sequência para condução de atividades. As categorias são apresentadas na tabela 4.

Tabela 4 - Dimensão Desenho de Pesquisa: Objeto da Investigação

\begin{tabular}{c|c|c}
\hline Objeto da Investigação & Ocorrência & Percentual \\
\hline Qualidade & 9 & $20 \%$ \\
\hline Valor Empresarial & 9 & $20 \%$ \\
\hline Mercado & 8 & $18 \%$ \\
\hline Aprendizado & 6 & $14 \%$ \\
\hline Valor Pessoal & 6 & $14 \%$ \\
\hline Tecnologia & 3 & $7 \%$ \\
\hline Desempenho & 2 & $5 \%$ \\
\hline Metodologia & 1 & $2 \%$ \\
\hline
\end{tabular}

Fonte: dados da pesquisa, 2013. 
Por fim, o sujeito da investigação, o qual indica os indivíduos ou aqueles que representam bem a população da pesquisa, tendo a capacidade de captar a real heterogeneidade existente no universo (COSTA, 2011), pode ser agrupado em oito categorias, de acordo com a tabela 5.

Tabela 5 - Dimensão Desenho de Pesquisa: Sujeito da Investigação

\begin{tabular}{c|c|c}
\hline Sujeito da Investigação & $\begin{array}{c}\text { Ocorrên- } \\
\text { cia }\end{array}$ & Percentual \\
\hline Empresas & 17 & $39 \%$ \\
\hline Estudantes & 8 & $18 \%$ \\
\hline Consumidores & 8 & $18 \%$ \\
\hline Empregados & 3 & $7 \%$ \\
\hline Órgãos/Entidades & 3 & $7 \%$ \\
\hline Professores & 2 & $5 \%$ \\
\hline Países & 2 & $5 \%$ \\
\hline Gestores & 1 & $2 \%$ \\
\hline
\end{tabular}

Fonte: dados da pesquisa, 2013.

\subsection{Instrumento de Medida e Coleta dos Dados}

Na investigação científica, os instrumentos de pesquisa são utilizados para "ler a realidade". Por isso, o esforço do pesquisador está vinculado à produção de resultados verdadeiros, ou seja, aproximar-se ao máximo da realidade. Nas Ciências Sociais, essa busca é particularmente crítica, pois geralmente os fenômenos investigados medem o comportamento e a percepção dos indivíduos (HOPPEN; LAPOINTE; MOREAU, 1996).

Assim esta dimensão, busca mensurar as técnicas descritas nos artigos analisados, ao coletar os instrumentos empregados na pesquisa e os métodos utilizados para selecionar, determinar e verificar a amostra dos dados. Portanto, agrupou-se em dez subgrupos de investigação as técnicas utilizadas, ao concentrar-se nos aspectos relacionados ao tipo de instrumento, estratégia de aplicação e modo de coleta, sendo: 1) técnicas adotadas na etapa exploratória para a construção e refinamento do instrumento; 2) verificação da validade do conteúdo/face; 3) técnicas adotadas na etapa quantitativa para a obtenção dos dados; 4) tipos dos dados; 5) classificação genérica de respondentes; 6) tamanho da amostra; 7) tipo da amostra; 8) taxa de resposta; 9) teste do viés; e, 10) caracterização da amostra sem comparação com a população e com comparação com a população. Os resultados são apresentados na tabela 6 . 


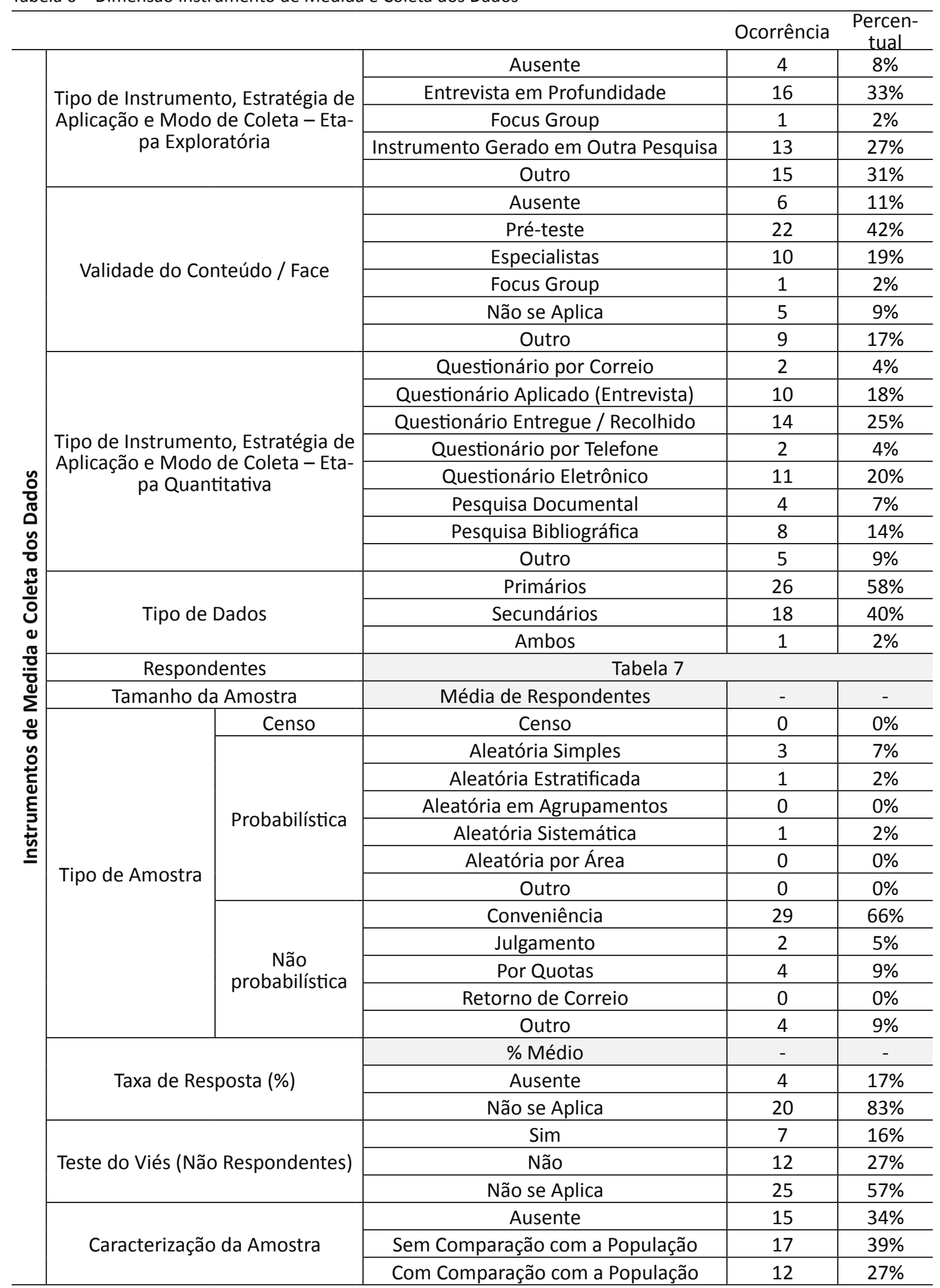

Fonte: dados da pesquisa, 2013. 
A classificação genérica de respondentes dos artigos analisados pode ser agrupada em sete categorias, as quais são apresentadas na tabela 7 .

Tabela 7 - Dimensão Instrumento de Medida e Coleta dos Dados: Respondentes

\begin{tabular}{c|c|c}
\hline Respondentes & Ocorrência & Percentual \\
\hline Consumidores & 11 & $25 \%$ \\
\hline Estudantes & 10 & $23 \%$ \\
\hline Gerentes & 9 & $20 \%$ \\
\hline Empregados & 6 & $14 \%$ \\
\hline Dados Secundários & 4 & $9 \%$ \\
\hline Professores & 2 & $5 \%$ \\
\hline Outros & 2 & $5 \%$ \\
\hline
\end{tabular}

Fonte: dados da pesquisa, 2013.

Este agrupamento de dados permite relacionar os respondentes do questionário de pesquisa com o sujeito da investigação, conforme anteriormente apresentado na tabela 5 . Então, nos estudos analisados confirmou-se que o respondente é o elemento que caracteriza o sujeito da pesquisa e uma vez alcançado, possibilitou aos pesquisadores avaliarem o proposto no objetivo dos trabalhos analisados.

\subsection{Validade dos Construtos}

O fato de uma medida ser confiável não quer dizer que seja uma medida válida. Porém, uma medida válida é necessariamente confiável. A validade de construto refere-se ao grau em que uma medida confirma hipóteses de semelhança com outras medidas do mesmo construto (convergente) e confirma ainda sua diferença para medidas de outros construtos (discriminante). A validade, então, consiste na verificação do comportamento da medida em relação a determinada expectativa teórica associada à definição do construto (COSTA, 2011). Por isso, esta dimensão identifica a utilização de métodos para verificar o comportamento da medida adotada em relação a uma determinada expectativa teórica, a fim de obter a confirmação estatística da relação entre os dados e a suposição de origem. Os resultados são apresentados na tabela 8.

Tabela 8 - Dimensão Validade dos Construtos

\begin{tabular}{c|c|c|c|c}
\hline \multicolumn{2}{c}{} & \multicolumn{2}{c}{ Ocorrência } & \multicolumn{2}{c}{ Percen- } \\
tual
\end{tabular}

Fonte: dados da pesquisa, 2013.

A validade de um construto está relacionada à questão do que o estudo, por meio do instrumento de pesquisa, está realmente medindo, e é avaliada em duas dimensões. Primeiro, a questão da confiabilidade, sendo a técnica mais utilizada nos estudos analisados o Alpha de 
Cronbach, porém os pesquisadores utilizam-se de outras técnicas, inclusive em alguns trabalhos, foi identificado que a verificação de confiabilidade utiliza simultaneamente o Alpha de Cronbach com outras técnicas estatísticas. Ressalta-se que em 4 trabalhos não foi possível identificar a utilização de alguma técnica de verificação de confiabilidade dos indicadores. Segundo, a questão da validade, a qual pode ser analisada por meio da validade convergente (consistência sob diferentes enunciados) e também, por meio da validade discriminante (diferença em relação a outras medidas de construtos diferentes). Verifica-se neste aspecto, que diversos trabalhos não confirmaram a validade dos seus indicadores. Isto, sem dúvidas pode levar o modelo teórico em investigação a contestação, visto que os indicadores estatisticamente não se mostraram significantes, o que é sempre crucial para os fundamentos teóricos e a interpretação dos resultados empíricos (HAIR; RINGLE; SARSTEDT, 2011).

\subsection{Análise dos Dados}

Quanto à análise de dados, deve-se julgar a pertinência e a qualidade de aplicação dos métodos utilizados. Basta lembrar aqui que qualquer que seja o método utilizado, o rigor e a honestidade são necessários durante a análise de dados (HOPPEN; LAPOINTE; MOREAU, 1996). Assim, esta dimensão, identifica os testes estatísticos que foram utilizados para processar, validar e interpretar os dados. Os resultados são apresentados na tabela 9.

Tabela 9 - Dimensão Análise dos Dados

\begin{tabular}{|c|c|c|c|c|}
\hline & & & Ocorrência & $\begin{array}{c}\text { Percen- } \\
\text { tual }\end{array}$ \\
\hline \multirow{12}{*}{ 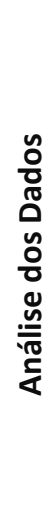 } & \multirow{12}{*}{ Tipos de Análise (Quais) } & Fatorial & 25 & $27 \%$ \\
\hline & & Média & 14 & $15 \%$ \\
\hline & & Desvio Padrão & 12 & $13 \%$ \\
\hline & & Qui-quadrado & 9 & $10 \%$ \\
\hline & & Regressão (Múltipla) & 6 & $6 \%$ \\
\hline & & Regressão (Correlação) & 4 & $4 \%$ \\
\hline & & Mediana & 3 & $3 \%$ \\
\hline & & Frequência & 1 & $1 \%$ \\
\hline & & Moda & 1 & $1 \%$ \\
\hline & & Discriminante & 1 & $1 \%$ \\
\hline & & Anova/Teste $\mathrm{t}$ & 1 & $1 \%$ \\
\hline & & Outro & 16 & $17 \%$ \\
\hline
\end{tabular}

Fonte: dados da pesquisa, 2013.

\subsection{Apresentação dos Resultados}

Os resultados de uma pesquisa devem ser apresentados de tal forma, que o leitor tenha condição de fazer a ligação com a teoria que serviu ao desenvolvimento do modelo e das hipóteses. Assim, esta dimensão identifica a qualidade e a credibilidade da pesquisa ao explicar o significado dos resultados, apresentar as inferências válidas e a capacidade de generalizar os resultados obtidos para a população, além de sugerir linhas de investigação para estudos futuros e apontar a instrumentação da teoria aplicada na prática. Os resultados são apresentados na tabela 10. 
Tabela 10 - Dimensão Apresentação dos Resultados

\begin{tabular}{|c|c|c|c|c|}
\hline & & & Ocorrência & Percentual \\
\hline \multirow{10}{*}{ 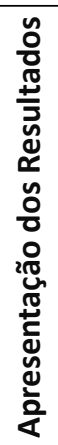 } & \multirow{2}{*}{ Validade Externa } & Sim & 18 & $41 \%$ \\
\hline & & Não & 26 & $59 \%$ \\
\hline & \multirow{2}{*}{ Validade Nomológica } & Sim & 12 & $27 \%$ \\
\hline & & Não & 32 & $73 \%$ \\
\hline & \multirow{2}{*}{ Limites do Estudo } & Sim & 31 & $70 \%$ \\
\hline & & Não & 13 & $30 \%$ \\
\hline & \multirow{2}{*}{ Recomendações para Pesquisas } & Sim & 37 & $84 \%$ \\
\hline & & Não & 7 & $16 \%$ \\
\hline & \multirow{2}{*}{ Recomendações Aplicadas } & Sim & 21 & $48 \%$ \\
\hline & & Não & 23 & $52 \%$ \\
\hline
\end{tabular}

Fonte: dados da pesquisa, 2013.

Nota-se que os resultados da pesquisa podem ser generalizados e aplicados a outras situações ou outras populações (validade externa) em 18 trabalhos. Também é possível verificar se a medida desenvolvida pelo pesquisador enquadra-se no sentido previsto pelo modelo teórico (validade nomológica) em 12 trabalhos. As limitações do estudo foram apresentadas em 31 trabalhos, por sua vez, 37 trabalhos apresentaram recomendações para pesquisas futuras, o que pode auxiliar outros pesquisadores que desejem avançar o estudo em questão. Por fim, 21 trabalhos tiveram suas recomendações teóricas aplicadas explicando as consequências dos resultados na prática.

\section{CONSIDERAÇÕES FINAIS}

Estudos que abrangem a questão da análise de artigos ocasionam o surgimento de uma produção científica capaz de reconhecer os pontos fortes e fracos desses artigos, bem como de colaborar para a melhoria da qualidade das pesquisas em diferentes subáreas (CORDEIRO et al., 2014). Nesse sentido, com intuito de lançar um olhar crítico sobre a produção acadêmica, esta pesquisa movimentou-se na recuperação das informações de artigos científicos que adotaram a Modelagem de Equações Estruturais (MEE), com a finalidade de mensurar os critérios utilizados nestes trabalhos ao capturar as dimensões de qualidade necessárias para tratamento dos dados e relato dos resultados em estudos quantitativos.

Uma vez, que o mapeamento bibliográfico permite uma visualização com certa precisão da produção científica (ANDRADE-JÚNIOR; CERANTO, 2013), verificou-se que a realização de pesquisas na área de Administração e Sistemas de Informação de base quantitativa com auxílio da Modelagem de Equações Estruturais é incipiente ( $~ 0,44 \%$ do universo de artigos), mas tem se mostrado constante ao longo do período analisado. Isso significa, que os métodos quantitativos têm sido mais bem compreendidos pelos pesquisadores ao apontar para a pesquisa que relaciona teoria e prática, para tentar explicar fenômenos complexos decorrentes de analises fundamentadas em indicadores extraídos do "mundo real".

Pela análise das dimensões avaliadas, nota-se que os artigos analisados por este trabaIho, apresentaram rigor moderado na preparação e instrumentalização das pesquisas, ao considerar na sua maioria os itens de qualidade esperados em uma pesquisa quantitativa, quando se confronta a descrição conceitual dos procedimentos e técnicas utilizadas dos artigos analisados com a metodologia de pesquisa adaptada de Hoppen, Lapointe e Moreau (1996), a qual foi utilizada neste trabalho. Por isso, é essencial questionar no começo da preparação de uma pesquisa, a pertinência do método escolhido, especialmente, verificar se existe uma adequação entre o método a ser utilizado e o problema de pesquisa definido. 
Acredita-se, que a principal limitação desta pesquisa, prende-se ao fato da quantidade de periódicos e artigos analisados. Também pode-se assumir como outra limitação do estudo, o intervalo temporal das publicações. Entretanto, superadas as limitações, acredita-se que esse tipo de pesquisa, oferece informações a respeito da qualidade do que é produzido no país e contribui para a compreensão e maior consistência da academia, já que pode incentivar reflexões críticas por parte dos autores, professores, alunos, eventos, periódicos, etc.

A principal contribuição deste estudo é orientar novas pesquisas sobre os aspectos que devem ser considerados durante o processo de construção de um estudo quantitativo, principalmente, ao apresentar as discussões dos estudos com a MEE, visto que ao familiarizar novos pesquisadores com os pontos de sustentação do método, é possível promover uma produção acadêmica de qualidade com resultados que corroboram para a evolução da Ciência. Pois, ao registrar um panorama, este trabalho mensurou, agrupou e apresentou os critérios utilizados nos estudos, que adotaram para a análise dos dados a MEE de modo a capturar todas as dimensões da qualidade esperadas em uma pesquisa quantitativa, ao identificar os elementos cuja fragilidade ou inexistência prejudicam a compreensão dos resultados de um estudo.

Finalmente, como sugestão para pesquisas futuras, ampliar o corte temporal para mais de cinco anos e a seleção de artigos para outras fontes de dados, como por exemplo, o banco de artigos do Encontro da Associação Nacional de Pós-Graduação e Pesquisa em Administração (EnANPAD), pode permitir a comparação dos resultados deste estudo, para assimilar a evolução e as dimensões de qualidade dos estudos quantitativos que relataram seus resultados com o apoio da Modelagem de Equações Estruturais na área de Administração e Sistemas de Informação.

\section{REFERENCIAS}

ANDRADE-JÚNIOR, P. P. D.; CERANTO, F. A. A. Um retrato bibliométrico da produção científica brasileira sobre gestão da Tecnologia e da Inovação no período 2001-2011. Revista de Administração da UFSM, Santa Maria, v. 6, n. 4, p. 708-719, dez., 2013.

BIDO, D. D. S. et al. Qualidade do Relato dos Procedimentos Metodológicos em Periódicos Nacionais na Área de Administração de Empresas: o caso da modelagem em equações estruturais nos periódicos nacionais entre 2001 e 2010. Organizações \& Sociedade, Salvador, v.19, n. 60, p. 125-144, jan./mar. 2012.

CODES, A. L. M. D. Modelagem de Equações Estruturais: um método para a análise de fenômenos complexos. Caderno CRH UFBA, Salvador, v. 18 , n. 45 , p. $471-484$, set./dez. 2005.

CORDEIRO, R. A. et al. Pesquisa quantitativa em finanças: uma análise das técnicas estatísticas utilizadas por artigos científicos publicados em periódicos qualificados no triênio 2007 a 2009. Revista de Administração da UFSM, Santa Maria, v. 7, n. 1, p. 117-134, mar., 2014.

COSTA, J. F. D. Mensuração e desenvolvimento de escalas: aplicações em administração. Rio de Janeiro: Ciência Moderna, 2011.

GOSLING, M.; GONÇALVES, C. A. Modelagem por Equações Estruturais: conceitos e aplicações. Revista de Administração FACES, Belo Horizonte, v. 2, n. 2, p. 83-95, ago./dez. 2003.

HAIR JR., J. F. et al. A Primer on Partial Least Squares Structural Equation Modeling (PLSSEM). Thousand Oaks: Sage, 2013.

HAIR, J. F.; RINGLE, C. M.; SARSTEDT, M. PLS-SEM: Indeed a Silver Bullet. Journal of Marketing Theory and Practice, v. 19, n. 2, p. 139-151, 2011.

HOPPEN, N.; LAPOINTE, L.; MOREAU, E. Um guia para a avaliação de artigos de pesquisa em sistemas de informação. REAd. Revista 
Eletrônica de Administração, Porto Alegre, ed. 3, v. 2, n. 2, set./out, 1996.

MALHOTRA, N. K. Pesquisa de Marketing: uma orientação aplicada. Tradução de Lene Belon Ribeiro; Monica Stefani. 6. ed. Porto Alegre: Bookman, 2012.

MENDES, E. L. Uma Metodologia para Avaliação da Satisfação do Consumidor com os Serviços Prestados pelas Distribuidoras. Tese de Doutorado. Pontifícia Universidade Católica do Rio de Janeiro - PUC Rio. Rio de Janeiro. 2006.

SOLA, G. L.; BONACIM, C. A. G. Avaliação bibliométrica de periódicos brasileiros: contrastando a metodologia Qualis-CAPES com o modelo de Krzyzanowski e Ferreira (1998). Revista de Administração da UFSM, Santa Maria, v. 6, n. 1, p. 55-70, jan./mar., 2013.

ZWICKER, R.; SOUZA, C. A. D.; BIDO, D. D. S. Uma revisão do Modelo do Grau de Informatização de Empresas: novas propostas de estimação e modelagem usando PLS (partial least squares). In: ENCONTRO DA ASSOCIAÇÃO NACIONAL DE PÓS-GRADUAÇÃO E PESQUISA EM ADMNISTRAÇÃO, 32., 2008, Rio de Janeiro. Anais... Rio de Janeiro: ANPAD. 2008.

\section{APÊNDICE 1 - ARTIGOS ANALISADOS}

ABBADE, E. B.; ZANINI, R. R.; SOUZA, A. M. Orientação para Aprendizagem, Orientação para Mercado e Desempenho Organizacional: Evidências Empíricas. Revista de Administração Contemporânea, v. 16, n. 1, p. 118-136, jan./ fev. Rio de Janeiro, 2012.

ALMEIDA, S. O. et al. Os Efeitos da Participação em Comunidades Virtuais de Marca no Comportamento do Consumidor: Proposição e Teste de um Modelo Teórico. Revista de Administração Contemporânea, v. 15, n. 3, p. 366-391, maio/jun. Curitiba, 2011.
ANDRADE, D. M.; BRANDÃO, M. M. Boca a Boca Eletrônico como Forma de Retaliação: uma Aplicação da Modelagem de Equações Estruturais para Determinar o Comportamento Pós-Reclamação de Consumidores Insatisfeitos/Ofendidos. Revista do Mestrado em Administração e Desenvolvimento Empresarial, v. 16, n. 1, p. 24-44, jan./abr. Rio de Janeiro, 2012.

ARAUJO, B. F. V. B.; BILSKY, W.; MOREIRA, L. M. C. O. Valores pessoais como antecedentes da adaptação transcultural de expatriados. Revista de Administração Mackenzie, v. 13, n. 3, p. 69-95, maio/jun. São Paulo, 2012.

BIDO, D. S. et al. Articulação entre as aprendizagens individual, grupal e organizacional: um estudo no ambiente industrial. Revista de Administração Mackenzie, v. 11, n. 2, p. 68-95, mar./abr. São Paulo, 2010.

BOEHE, D. M. The Influence of Coordination Mechanisms on New Product Development in MNC Subsidiaries. Brazilian Administration Review, v. 7, n. 1, p. 79-97, Jan./Mar. Curitiba, 2010.

CARNEIRO, J.; ROCHA, A.; SILVA, J. F. Determinants of Export Performance: a Study of Large Brazilian Manufacturing Firms. Brazilian Administration Review, v. 8, n. 2, p. 107-132, Apr/Jun. Curitiba, 2011.

CARNEIRO, J.; ROCHA, A.; SILVA, J. F. Proposal of a Validation Framework for a New Measurement Model and its Application to the Export Performance Construct. Brazilian Administration Review, v. 6, n. 4, p. 331-353, Oct./Dec. Curitiba, 2009.

COSTA, A. C. F.; SOUZA, S. S.; SILVA, L. C. T. Investigação sobre a satisfação do usuário dos serviços prestados pelo metrô de São Paulo: um estudo exploratório, descritivo e ilustrativo com a utilização do modelo de equações estruturais. Revista de Gestão, v. 15, n. especial, p. 93-108. São Paulo, 2008. 
DAVIDOVITSCH, L.; CALDAS, L. F.; SILVA, J. F. Estruturação das redes de fast food: um estudo empírico sobre os antecedentes do franchising e suas implicações sobre o desempenho das firmas. Revista de Administração, v. 44, n. 4, p. 299-312, out./nov./dez. São Paulo, 2009.

DAVIDOVITSCH, L.; SILVA, J. F. Algumas implicações da percepção dos Valores simbólicos das roupas: gênero Masculino em foco. Revista de Administração Mackenzie, v. 11, n. 1, p. 150-173, jan./fev. São Paulo, 2010.

DEMO, G. etal. Human Resources Management Policies and Practices Scale (HRMPPS): Exploratory and Confirmatory Factor Analysis. Brazilian Administration Review, v. 9, n. 4, p. 395-420, Oct./Dec. Rio de Janeiro, 2012.

FABRIS, C.; NETO, P. J. S.; TOALDO, A. M. M. Evidências Empíricas da Influência da Família, Mídia, Escola e Pares nos Antecedentes e no Comportamento de Separação de Materiais para a Reciclagem. Revista de Administração Contemporânea, v. 14, n. 6, p. 1134-1157, nov./dez. Curitiba, 2010.

FILHO, C. G.; GONÇALVES, C. A.; SOUKI, G. Q. Valor das Marcas (Brand Equity) para o Consumidor: Desenvolvimento e Validação de um Instrumento de Mensuração no Setor Automotivo. Revista Eletrônica de Ciência Administrativa, v. 8 , n. 1 , p. 106-118, maio. Campo Largo, 2009.

FREGA, J. R.; LEMOS, I. S.; SOUZA, A. Atributos Relevantes de Destinos Turísticos na Percepção de Internautas Brasileiros. Turismo em Análise, v. 21, n. 2, p. 271-296, ago. São Paulo, 2010.

GARRIDO, I. L. et al. A escala Experf e os modos de entrada: uma proposta de mensuração de desempenho internacional em empresas brasileiras. Revista de Administração e Contabilidade Base, v. 6, n. 4, p. 312-327, nov./ dez. São Leopoldo, 2009.

GODOY, A. S. et al. O desenvolvimento das competências de alunos formandos do curso de Administração: um estudo de modelagem de equações estruturais. Revista de Administração, v. 44, n.3, p. 265-278, jul./ ago./set. São Paulo, 2009.

HILDEBRAND, D. F. N. et al. Consumer-company Identification: Development and Validation of a Scale. Brazilian Administration Review, v. 7, n. 3, p. 276-293, Jul./Sep. Curitiba, 2010.

HORTA, P.; DEMO, G.; ROURE, P. Políticas de Gestão de Pessoas, Confiança e Bem-estar: Estudo em uma Multinacional. Revista de Administração Contemporânea, v. 16, n. 4, p. 566-585, jul./ago. Rio de Janeiro, 2012.

IFINEDO, P. Drivers of e-government maturity in two developing regions: focus on Latin America and sub-Saharan Africa. Journal of Information Systems and Technology Management, v. 9, n.1, p. 5-22, Jan./Apr. São Paulo, 2012.

LADEIRA, W. J.; SONZA, I. B.; BERTE, R. S. Antecedentes da satisfação no setor público: um estudo de caso na prefeitura de Santa Maria (RS). Revista de Administração Pública, v. 46 , n. 1, p. 71-91, jan./fev. Rio de Janeiro, 2012.

LAMEIRA, V. J. et al. Governança, Risco e Desempenho das companhias abertas brasileiras. Revista de Economia e Administração, v. 9, n. 1, p. 17-76 jan./mar. São Paulo, 2010.

LÖBLER, M. L. et al. As influências na intenção de uso dos sistemas de informação: uma abordagem entre a teoria de estilos cognitivos de Kirton e a teoria Unificada de Aceitação e uso da tecnologia. Revista de Administração e Inovação, v. 8, n. 2, p. 55-81, abr./jun. São Paulo, 2011.

MACIEL, C. O.; CAMARGO, C. Comprometimento, Satisfação e Cooperação no Trabalho: Evidências da Primazia dos Aspectos Morais e das Normas de Reciprocidade Sobre o Comportamento. Revista de Administração 
Contemporânea, v. 15, n. 3, p. 433-453, maio/ jun. Curitiba, 2011.

MARCON, R.; MELLO, R. B.; ALBERTON, A. Instrumental Stakeholder Theory in Turbulent Environments: An Empirical Testing using Political and Social Donations. Brazilian Business Review, v. 5, n. 3, p. 275-293, Sep./ Dec. Vitória, 2008.

MENDES-DA-SILVA, W.; BIDO, D. S.; FORTE, D. Atributos determinantes do desempenho do professor de Finanças: estudo empírico. Revista de Economia e Administração, v. 10, n. 3, p. 393-411, jul./set. São Paulo, 2011.

MILACH, F. T.; HUPPES, D.; VIEIRA, K. M. Equações estruturais aplicadas à satisfação dos alunos: um estudo no curso de Ciências Contábeis da Universidade Federal de Santa Maria. Revista Contabilidade \& Finanças, v. 19, n. 48, p. 65-79, set./dez. São Paulo, 2008.

MILAN, G. S. et al. A prática relacional entre provedor de serviços e clientes: comparação entre um modelo teórico e um modelo rival. Revista de Administração e Contabilidade Base, v. 7, n. 1, p. 42-54, jan./mar. São Leopoldo, 2010.

MILAN, G. S.; TONI, D. A construção de um modelo sobre a retenção de clientes e seus antecedentes em um ambiente de serviços. REAd. Revista Eletrônica de Administração, v. 72, n. 2, p. 433-467, maio/ago. Porto Alegre, 2012.

PAIVA, E. L.; GAVRONSKI, I.; D'AVILA, L. C. The Relationship between Manufacturing Integration and Performance from an ActivityOriented Perspective. Brazilian Administration Review, v. 8, n. 4, p. 376-394, Oct./Dec. Curitiba, 2011.

POPADIUK, S.; SANTOS, C. R. Fatores de influência na adoção da metodologia de gestão de projetos em TI: uma comparação entre usuários e potenciais usuários mediante o uso do SMARTPLS. REAd. Revista Eletrônica de Administração, e. 59 , v. 14 , n. 1, p. 1-25, jan./abr. Porto Alegre, 2008.

RIBEIRO, J. A.; VEIGA, R. T. Proposição de uma escala de consumo sustentável. Revista de Administração, v. 46, n. 1, p. 45-60, jan./fev./ mar. São Paulo, 2011.

SANTOS, C. P.; FERNANDES, D. V. H. Perceptions of Justice after Recovery Efforts in Internet Purchasing: the Impact on Consumer Trust and Loyalty toward Retailing Sites and Online Shopping in General. Brazilian Administration Review, v. 8, n. 3, p. 225-246, Jul./Sep. Curitiba, 2011.

SCHMIDT, S.; BOHNENBERGER, M. C. Perfil Empreendedor e Desempenho Organizacional. Revista de Administração Contemporânea, v. 13, n. 3, p. 450-467, jul./ago. Curitiba, 2009.

SOBRAL, F. J. B. A. Relação dinâmica entre confiança, competitividade e o processo de tomada de decisão ética em negociação. Revista de Administração de Empresas, v. 9, n. 2, jul./dez. São Paulo, 2010.

SOBRAL, F. J. B. A. O julgamento moral de dilemas éticos em negociação. Revista de Administração Mackenzie, v. 10, n. 5, p. 4-27, set./out. São Paulo, 2009.

SOUSA, W. H.; IGAMI, M. P. Z.; BIDO, D. S. R\&D Management and the Stokes Diagram: An Exploratory Study. Journal of Technology Management \& Innovation, v. 4, n. 4, p. 95109. Santiago/Chile, 2009.

THOMAZ, J. C.; BRITO, E. P. Z. Reputação Corporativa: Construtos Formativos e Implicações para a Gestão. Revista de Administração Contemporânea, v. 14, n. 2, p. 229-250, mar./abr. Curitiba, 2010.

TIBOLA, J. A. et al. Antecedentes da permanência de estudantes de uma instituição de ensino superior: um modelo confirmatório. Revista Alcance, v. 19 , n. 1, p. 83-100, jan./ mar. Biguaçu, 2012. 
VIEIRA, K. M.; BECKER, J. L. Modelagem de Equações Estruturais Aplicada à Reação a Bonificações e Desdobramentos: Integrando as Hipóteses de Sinalização, Liquidez e Nível Ótimo de Preços. Revista Brasileira de Finanças, v. 9, n. 1, p. 69-104, jan./mar. Rio de Janeiro, 2011.

VIEIRA, P. R. C.; TROCOLI, I, R, SILVA, J. M. C. (In memoriam). A Relevância do Corpo Docente na Qualidade Percebida em Serviço de Ensino Superior no Brasil: um Estudo com Modelagem de Equações Estruturais. Revista Economia \& Gestão, v. 11, n. 26, maio/ago. Belo Horizonte, 2011.

VIEIRA, V. A.; APPIO, J. O Impacto da Conexidade no Comportamento do Consumidor em Relação aos Programas de Televisão. Revista de Administração Contemporânea, v. 14, n. 4, p. 703-721, jul./ago. Curitiba, 2010.

VIEIRA, V. A. A Prototype Design for Enhancing Customer Trust in Online Payments. Journal of Computer Science, v. 5, n. 2, p. 1034-1041. Adelaide/Australia, 2009.

VIEIRA, V. A. Is the Need to Evaluate a Real Consumers' Need? Brazilian Administration Review, v. 6, n. 2, p. 153-172, Apr./Jun. Curitiba, 2009. 